\title{
Solving the heart curve with geogebra
}

\author{
Ida Nuraida $^{*}$, Hamdan Sugilar, Rahayu Kariadinata, Juariah Juariah, and Nunung Sobarningsih \\ UIN Sunan Gunung Djati Bandung, Bandung, Indonesia
}

\begin{abstract}
Heart symbols are used as expressions of love. There are several mathematical explanations that can produce a curve in the shape of a heart symbol, the most famous of which is cardioid. Graphs that look exotic like cardioid and limacon can be solved using polar coordinates. Polar equations $r=a \pm b \cos \theta$ and $r=a \pm b \sin \theta$, with $a$ and $b$ positive are called limacon curves, for the special case $a=b$ is called the cardiod. Computer technology is an alternative that can be used to help this. One of the computer programs that can be utilized as a medium of mathematics learning especially in drawing the heart curve is GeoGebra. GeoGebra is a dynamic mathematical software that combines geometry, algebra, and calculus. GeoGebra program serves as a learning medium that provides a visual experience to students in interacting with the concept of polar coordinates in solving the problem of the heart curve.
\end{abstract}

\section{Introduction}

In the discussion of the calculus of the heart curve is often called the cardiod. Cardiod itself is taken from the Greek "kardia" which means is "heart" [1]. Drawing the heart curve can be done manually, but it can also be done with the help of software. GeoGebra is a dynamic mathematical software that combines geometry, algebra, and calculus. This software was developed for the process of teaching mathematics at school by Markus Hohenwarter in 2001 at the University of Florida Atlantic.

GeoGebra provides a visualization experience and dynamics of geometric shapes, so that learning is felt more easily and appealing to teachers and students [2]. GeoGebra also has a positive impact on student achievement, the students have a positive perception on GeoGebra in terms of enthusiasm, confidence, and motivation [3]. GeoGebra is also very useful as a medium of learning mathematics with various activities, namely as a medium of demonstration and visualization, used as a tool for construction and as a tool of the process of discovery [4].

Geogebra has also been widely used to design various applets of calculus concepts [5]. GeoGebra as a mathematical software has enabled students to learn the concept of symmetry better. Students can visualize the concept of symmetry by integrating computers into mathematics [6]. Skills in GeoGebra and Critical Thinking as a reflective tool can develop students' understanding of probabilities [7]. Another study shows that the use of GeoGebra can improve student performance in learning Coordinate Geometry [8]

[9] conducted a study that explored students' thinking about polar coordinate systems. Students experience difficulties when they have to switch from cartesian coordinate to polar coordinate. Therefore, to understand a topic in polar coordinates requires a visualization tool that can help students understand this concept. In the previous research has been widely described the use of GeoGebra in several topics in learning mathematics. In this paper we will discuss the use of GeoGebra in solving the heart curve in polar coordinate.

\section{Literature Review}

The cardiovascular equation is a special form of the limacon equation, here is the equation

$$
\begin{aligned}
& r=a \pm b \sin \theta \\
& r=a \pm b \cos \theta
\end{aligned}
$$

with $a=b$ is called the cardiod equation [10]. The equation can be illustrated in cartesian coordinates. Figure 1 shows the equation $r=2+2 \sin \theta$. The cardiod equation can be represent in cartesian coordinates by shifting the upward sinus curve [11].

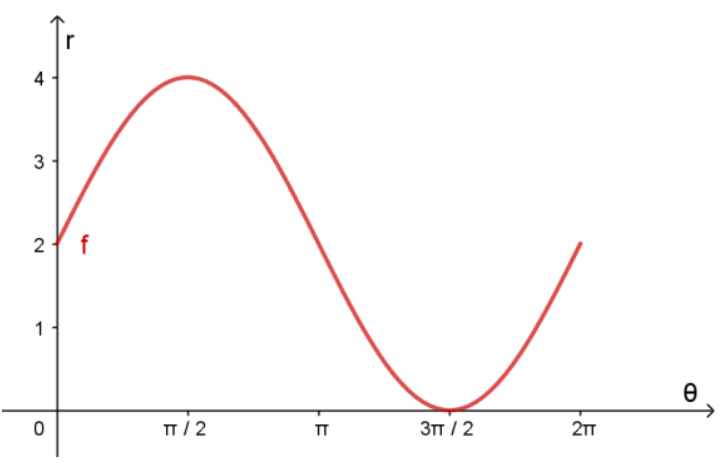

Fig. 1. $r=2+2 \sin \theta$ in Cartesian Coordinate.

The cardiac equation can also be represent in polar coordinates as shown in Figure 2 below

\footnotetext{
* Corresponding author: idanuraida@uinsgd.ac.id
} 


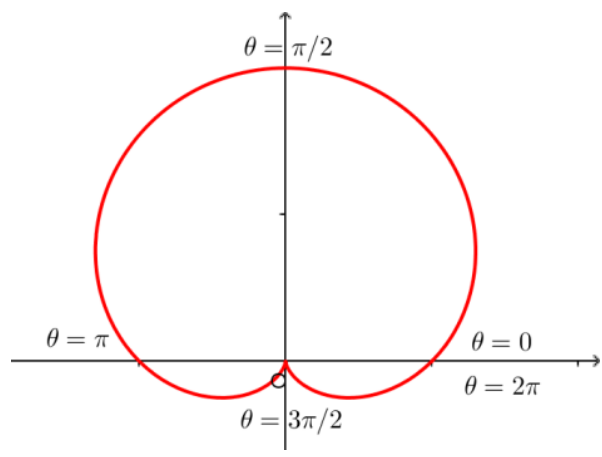

Fig. 2. $r=2+2 \sin \theta$ in Polar Coordinate.

When $\theta$ increases from 0 to $\frac{1}{2} \pi$, the $r$ value also increases from 2 to 4 units. As the value of $\theta$ increases from $1 / 2 \pi$ to $\pi$, the $r$ value decreases from 4 units to 2 units. Then when $\theta$ increases from $\pi$ to $\frac{3}{2} \pi$, then the $r$ value decreases from 2 units to 0 . And when $\theta$ increases from $\frac{3}{2} \pi$ to $2 \pi$, then $r$ increases again from 0 to 2 units. If $\theta$ increases beyond $2 \pi$ or less than 0 , then we will see the same curve again.

\section{Method}

Broadly speaking geogebra has parts, interface base of GeoGebra program, that is algebra view, toolbar, inputbar, CAS, spreadsheet, graphics 2D/3D.

To draw a heart curve done several steps, among them is

1. Converts the Cartesian coordinates to the polar coordinates. The characteristic position of a point in polar coordinates is expressed in the form $(r, \alpha)$, where $r$ is the distance between the polar axes at a given point.

2. Create a slider angle $(\alpha)$. Slider serves as a dynamic angle replacement variable.

3. Create equations of cardiodes by inputting equations in the input tool $(r)$.

4. Make a point with the notation $A=(r, \alpha)$ which will make a living footprint to form a heart curve.

5. Create a locus between point $\mathrm{A}$ and $\alpha$ slider. With this locus menu we will get the heart curve perfectly.

\section{Result and Discussion}

The results obtained in this discussion are some of the heart curves described in polar coordinates. There are 4 curves generated, taken from the cardiod equation. We will identify the characteristics of each of these equations by looking at the curves made in the GeoGebra software.

\subsection{Equation $r=2+2 \sin \theta$}

The card equation $r=2+2 \sin \theta \theta$ can be sketched in GeoGebra software using polar coordinates. As shown in Figure 3.

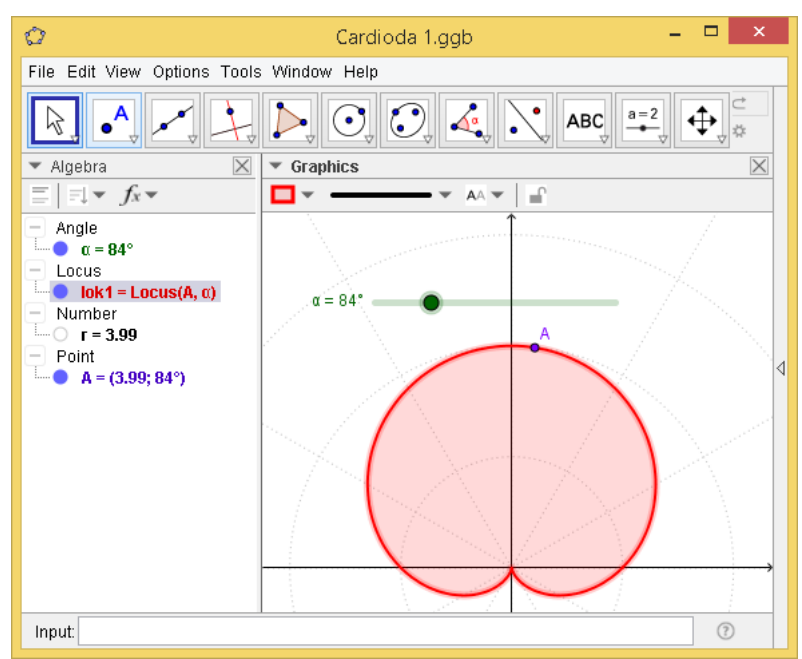

Fig. 3. Heart Curve with Equation $r=2+2 \sin \theta$.

The equation $r=2+2 \sin \theta$ can be constructed like a graph of the sinus function in Cartesian coordinates, where the change $\theta$ corresponds to a change in the value of $r$. The characteristic of the heart curve in this equation is seen facing downwards.

\subsection{Equation $r=2-2 \sin \theta$}

As with previous cardiac equations, the equation $r=$ $2-2 \sin \theta$ can also be made in GeoGebra software by using polar coordinates. As shown in Figure 4.

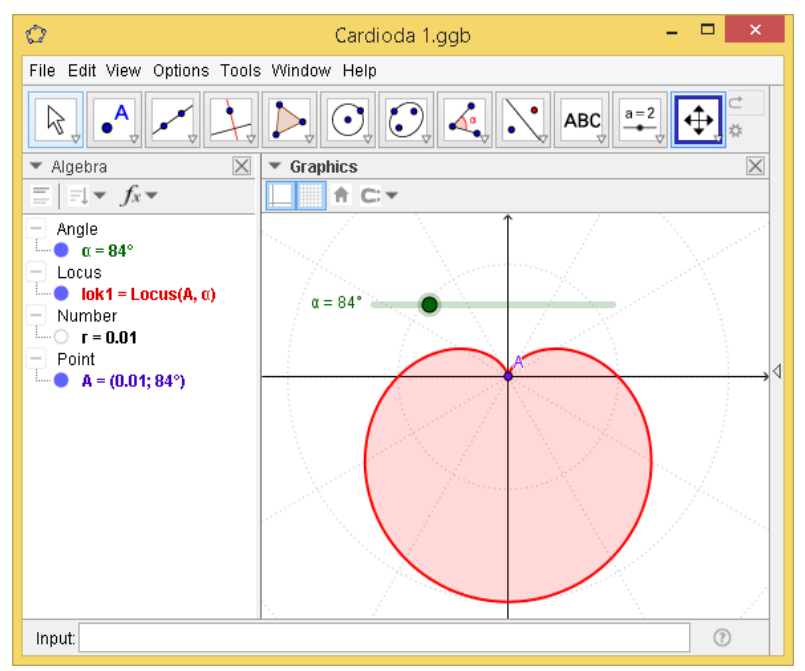

Fig. 4. Heart Curve with Equation $r=2-2 \sin \theta$.

If in the first equation the vertical heart-shaped curve, but facing downwards, the cardiac equation $r=2-$ $2 \sin \theta$ curve is seen facing upward. The characteristic of the sinus-shaped cardiac equation is the vertical-shaped kerva, where the curvature is facing up or down. 


\subsection{Equation $r=2+2 \cos \theta$}

Next is the equation kardioda shaped $r=2+2 \cos \omega \theta$. This equation can also form a horizontal-shaped heart curve as shown in Figure 5 below.

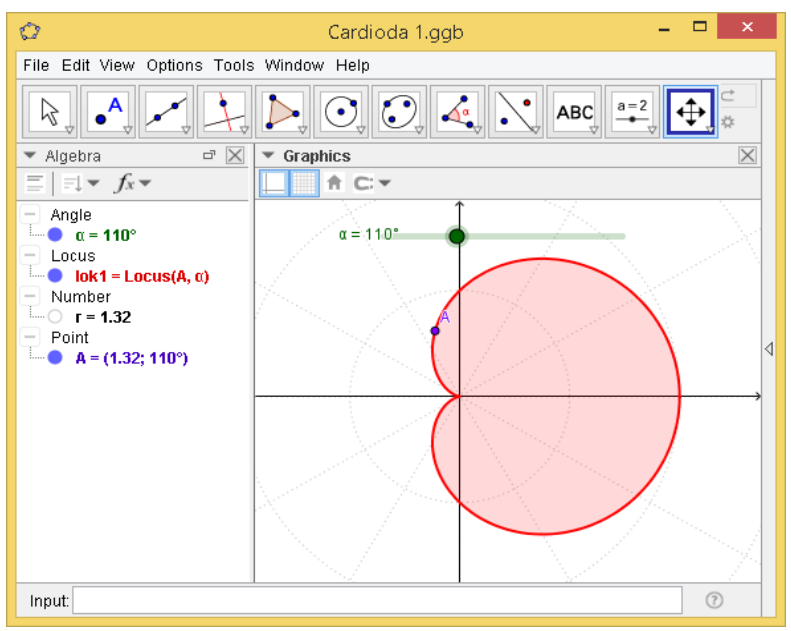

Fig. 5. Heart Curve with Equation $r=2+2 \cos \theta$.

In Figure 5, the equation of cardiod $r=2+2 \cos \theta$ can be formed into a heart curve. The heart curve that is formed is a curve that can be described as a graph of cosinus function. At $0 \leq \theta \leq \frac{1}{2} \pi$, the $r$ value changes from 2 units to 1 unit. At the interval $\frac{1}{2} \pi \leq \theta \leq \pi$, the cosinus chart also changes the value of $r$ from 1 unit to 0 units. At the $\pi \leq \theta \leq \frac{3}{2} \pi$ interval, the cosinus graph changes its value $r$ from 0 units to 1 unit. And lastly at $\frac{3}{2} \pi \leq \theta \leq 2 \pi$ interv intervals, the cosine graph changes $r$ value from 0 units to 2 units.

\subsection{Equation $r=2-2 \cos \theta$}

Last is the equation $r=2-2 \cos \theta \theta$. GeoGebra describes this equation as a heart-shaped curve as shown in Figure 6 below.

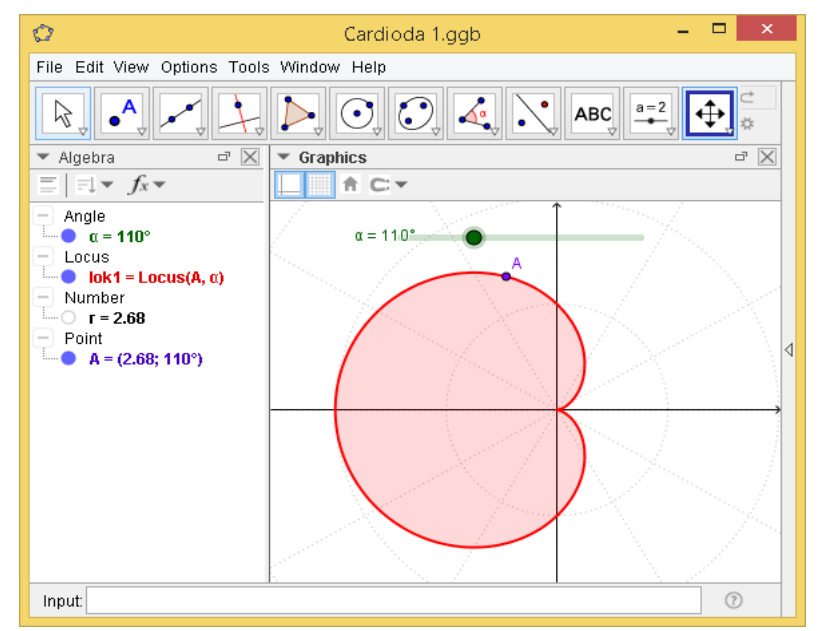

Fig. 6. Heart Curve with Equation $r=2+2 \cos \theta$.
The curve formed from the equation $r=2-2 \cos \theta$ is the cosinus equation as shown in Figure 5 which is horizontally facing to the left. Meanwhile, in Figure 6 the horizontal heart-shaped curve is facing to the right.

By looking at the results obtained this shows the difference in shape of the curves for each equation. The sinus-shaped equation produces a horizontal curve facing up or down, whereas in cosine-shaped equations the curve is the horizontal curve facing left or right.

\section{Conclusion}

By looking at the results obtained this shows the difference in shape of the curves for each equation. The sinus-shaped equation produces a horizontal curve facing up or down, whereas in cosine-shaped equations the curve is the horizontal curve facing left or right.

GeoGebra software can be used to assist in visualizing mathematical objects. In this case GeoGebra can help in understanding the shape of the haert curve, which is described in polar coordinates.

GeoGebra is able to demonstrate and visualize the forms of the heart curve. GeoGebra also helps in the process of concept construction in the formation of the heart curve. And lastly, GeoGebra is used as a tool of the discovery process. In this case GeoGebra is used as a tool for students to find a new concept, ie students are able to define the characteristics of each cardio equation identified through the image formed in GeoGebra.

\section{References}

1 Arseniy V. Akopyan, Geometry of the Cardioid, Am. Math. Mon., vol. 122, no. 2, p. 144, (2015).

2 Z. A. Reis and S. Ozdemir, "Using Geogebra as an information technology tool: Parabola teaching," Procedia - Soc. Behav. Sci., vol. 9, pp. 565-572, (2010).

3 N. Arbain and N. A. Shukor, The Effects of GeoGebra on Students Achievement, Procedia Soc. Behav. Sci., vol. 172, no. 2007, pp. 208-214, (2015).

4 A. Mahmudi, Membelajarkan Geometri dengan Program GeoGebra, Procedia - Natl. Semin. Math. Math. Educ. UNY, no. November, pp. 469-477, (2010).

5 M. Graciela Caligaris, M. E. Schivo, and M. R. Romiti, Calculus \& GeoGebra an Interesting Partnership, Procedia - Soc. Behav. Sci., vol. 174, pp. 1183-1188, 2015.

6 A. Akkaya, E. Tatar, and T. B. Kağizmanli, Using dynamic software in teaching of the symmetry in analytic geometry: The case of GeoGebra, Procedia - Soc. Behav. Sci., vol. 15, pp. 2540-2544, (2011).

7 E. Aizikovitsh-Udi and N. Radakovic, Teaching Probability by Using Geogebra Dynamic Tool and Implemanting Critical Thinking Skills, Procedia Soc. Behav. Sci., vol. 46, pp. 4943-4947, (2012).

8 R. A. Saha, A. F. M. Ayub, and R. A. Tarmizi, The 
effects of GeoGebra on mathematics achievement: Enlightening Coordinate Geometry learning, Procedia - Soc. Behav. Sci., vol. 8, no. 5, pp. 686693, (2010).

9 K. C. Moore, T. Paoletti, and S. Musgrave, Complexities in students' construction of the polar coordinate system, J. Math. Behav., vol. 36, pp. 135-149, (2014).

10 D. Varberg, E. J. Purcell, and S. E. Ridgon, Calculus, 9th ed. (United States of America: Pearson Education, Inc, 2007).

11 J. Stewart, Calculus, 8th ed. (USA: Cengage Learning, 2014). 